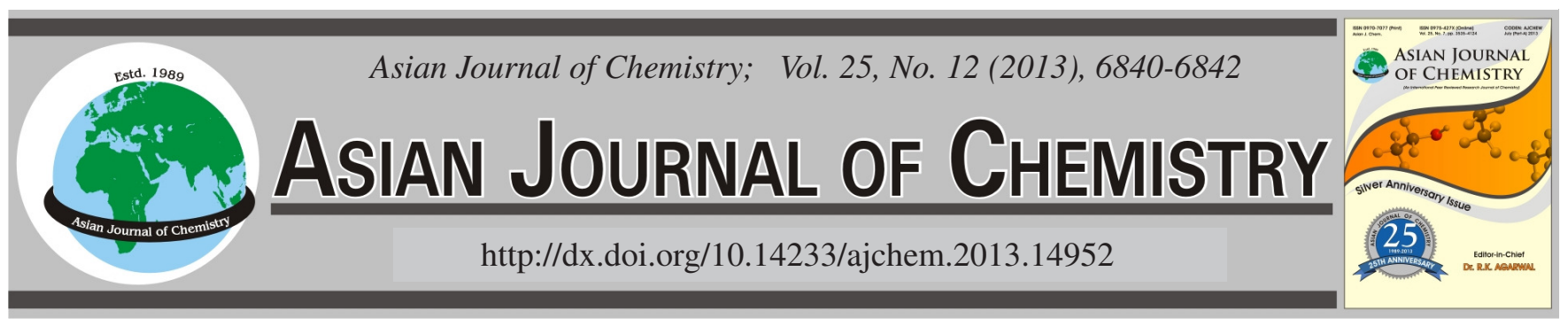

\title{
Reutilization Fast Hydrating Guar Fracturing Fluid
}

\author{
Zhanging Qu*, Xiaolong Li, Jie Zhang, Cheng Su and Shanshan Lin
}

College of Petroleum Engineering, China University of Petroleum, Qingdao 266580, P.R. China

*Corresponding author: Tel: +86 18654656599; E-mail: zjwd0603@ sina.com

(Received: 10 December 2012;

Accepted: 5 June 2013)

AJC-13583 According to the low efficiency of fracturing fluid preparing in fracturing operation, the recent rising price of guar raw materials, too
much drainage liquid after construction and the higher cost of treatment, fast hydrating guar fracturing fluid was developed and the
feasibility of regeneration was studied. Based on a series of experiments, such as the rate of dissolution determination of the guar, the
performance contrast between guar and conventional guar, gel breaking experiment and assessment of repeated crosslinking performance,
the fast hydrating guar performance and reutilization feasibility was studied. The experimental results show that, the fast hydrating guar
dissolved fast, with a good dispersion property and completely gel breaking performance, which can be as a thickening agent consisting
of fracturing fluid system with shear resistant performance, a certain reutilization performance, economy and environmental protection,
has a wide application prospect.

Key Words: Fast hydrating guar, Fracturing fluid, Reutilization, Gel breaking.

\section{INTRODUCTION}

Since the implementation of fracturing stimulation, the fracturing fluid of construction experienced three stages i.e., sesbania gum, guar gum and modified guar gum ${ }^{1-4}$, during which also carried out the exploration and field trials of oil-based fracturing fluid. However, because the water-based fracturing fluid is much cheaper and has an excellent performance and easy control performance and other characteristics, so it is currently used as the most widely fracturing fluid. Fracturing fluid as a fracture initiation and proppant carrying agent is an important part of the process of fracturing technology. Guar fracturing fluid is most commonly used and has a good proppant carrying capacity, fluid filtration control and rheological control features, but often have a low fluid efficiency and can not meet the needs of massive hydraulic fracture because of the slower dissolution rate of guar at the scene of the fracturing ${ }^{5-7}$.

In recent years, clean fracturing fluid is being applied more and more. On the one hand, it is more expensive, on the other hand, the gel breaking performance of the lower crude oil content reservoir is bad ${ }^{8,9}$. With the depression of clean fracturing fluid used in the U.S. and European markets, the major oil companies began turning a new direction to a conventional guar fracturing fluid, resulting in the recent the guar film price increasing promptly, the guar film price averaged $\$ 3,000 /$ ton in August 2011 and rose to \$6,000/ton by the end of 2011 and now nearly $\$ 15,000 /$ ton. Therefore, the study on the feasibility of the reutilization of fast hydrating guar fracturing fluid is urgently required.

\section{EXPERIMENTAL}

Principle: The fast hydrating guar has a high dissolution rate because the intramolecular hydrogen bond is weakened. The polymer particles need to undergo a swelling stage in the process of becoming soluble in aqueous solution, which includes the water molecules diffusing into the inside of the polymer particles, continuing to dissolve under the stirring and the inner layer polymer molecules swelling continuously, so the dissolution needs stirring constantly in the case of the generation of a 'fish eye'. Guar molecules, is generally believed that there are lots of intramolecular and intermolecular hydrogen bonds between the guar particles. It must first overcome guar intermolecular hydrogen bonds when it is dissolved in water, so the hydrogen bonds are one of the main reasons of slow dissolution of guar. From the view of molecular design and molecular structure, guar modified process should reduce the intermolecular hydrogen bonds, which can greatly reduce the guar plastic intermolecular hydrogen bonds by introducing the modifier ${ }^{10}$.

The water-soluble portion of the guar mainly $\beta-1,4$ glycosides keys coupled to D-mannose pyranose-based backbone, branched chain consisting of $\alpha-1,6$ glycosides key connection D-galacto-pyranosen on chain neutral multi-ortho-cis-hydroxy 
glycans. There are typically two forms in the hydrolysis process of the guar hydrolysis of the glycoside bond. One is a backbone of $\beta-1,4$ glycoside bond hydrolysis, so that the molecular weight and solution viscosity changed greatly. We obtain the short chain polymers by conventional polymer-guar main chain $\beta-1,4$ glycoside bond degradation. Addition of organic boron cross-linker, the short chain molecules are made to a highly dense polymer network structure. The fracturing fluid can easily be reversed by a $\mathrm{pH}$ value, the loss of crosslinking performance and completely degraded into shorter chain molecules before cross-linking. The formulation doesn't break the guar and maintains again cross-linking capability because it doesn't use conventional a breaker. The fracturing liquid can be recycled, reducing the potential environmental impact, the cost of oil and gas well stimulation ${ }^{11}$.

Methods: (1) Synthesis of the fast hydrating guar: Guar powder is dispersed in an ethanol/water solution, adding catalytic agent and modifier (the main purpose is to improve the dissolution rate of the guar polymer), dispersing agents (to improve the water dispersibility of the guar polymer), purged with nitrogen to achieve the deaerator purpose, heated to $60-70^{\circ} \mathrm{C}$, the reaction was stopped after $2-3 \mathrm{~h}$, neutralization solution with formic acid and then filtered and dried.

(2) Evaluation of dissolution rate: There is no standard method for the detection of guar polymer dissolution rate and we use the following method according to the characteristics of guar polymer: $19^{\circ} \mathrm{C}$, in a mixing device of distilled water (350 mL), Span80 (4 drops), in the $1000 \mathrm{~s}^{-1}$ stirring speed to join guar powder (powder ratio, $0.6 \%$ ) timing $45 \mathrm{~s}$ pour liquid NDJ-1 rotary viscometer, ascertain by measuring the apparent viscosity of solution of $511 \mathrm{~s}^{-1}$ in different time.

(3) Fracturing fluid performance evaluation: Evaluate the performance of guar fracturing fluid according to SY/T 5764-2007 “General Technical Specifications of Natural Plant Gum for Fracturing" and SY/T 5107-2005 "Water Base Fracturing Fluid Performance Evaluation Method".

(4) Repeated cross-linking properties: Determine the viscosity and rheology of soluble fast hydrating guar fracturing fluid before and after gel breaking and explore the feasibility of reutilization.

\section{RESULTS AND DISCUSSION}

Dissolution rate: To study on the dissolution rate of different guar powder, experiments use 6 guar powders: fast hydrating guar, Kunshan guar, JAG415 and JAG418 of Rodia company, highly dispersed HPG of Aqulon Company, CMHPG of BGRIMM, the experimental results are as shown in Fig. 1.

As can be seen from the Fig. 1: (1) The dissolution rate of guar power is quite different and the basic trend is to dissolve faster initially within 0-10 min, apparent viscosity changes, but essentially the same system viscosity at 10-20 min. So we should pay more attention to the viscosity changes in the first 10 min when study the dissolution rate of the guar, $20 \mathrm{~min}$ viscosity can see as the final viscosity of the system.

(2) JAG415 is a hydroxypropyl guar,while JAG418 is a carboxymethyl hydroxypropyl guar. Both have maximum viscosity and the initial dispersion is good, which also can't form 'fish eye' when dispersed in water, but the initial dissolution

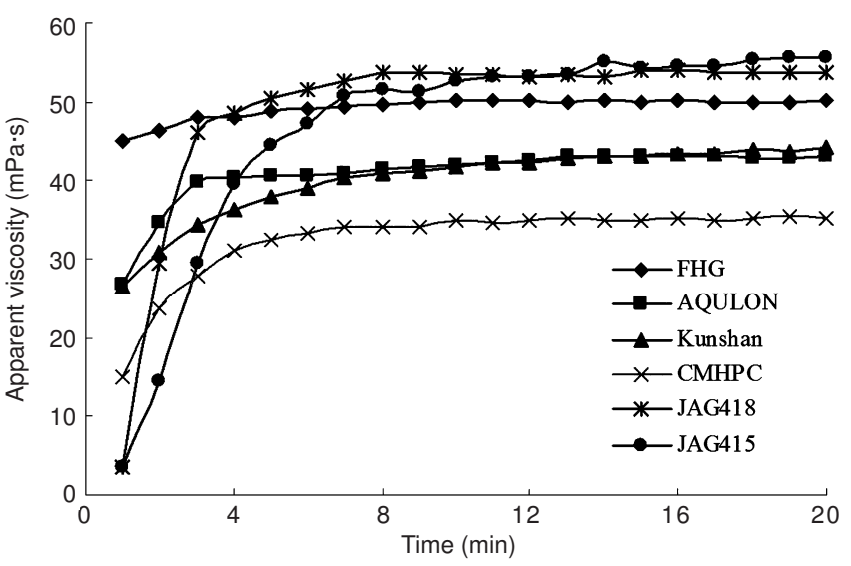

Fig. 1. Dissolution rate curve of six guar power

rate is low, since the rate is just high within 1-4 min and thereafter much slower.

(3) The final viscosity of Kunshan guar and CMHPG is lower and the latter is the lowest.

(4) The initial dissolution rate of fast hydrating guar is the highest and viscosity can reach $86 \%$ of the final viscosity in $1 \mathrm{~min}$, with a higher final viscosity close to the JAG415 and JAG418 viscosity level.

Performance evaluation: According to the SY/T 57642007 method, determination of different modified guar using performance, the experimental results are shown in Table-1.

As can be seen from the charts, JAG415 and JAG418 solution has the highest apparent viscosity, lowest water insoluble, good dispersion and also a big gap with others; $0.6 \%$ fast hydrating guar has a apparent viscosity of $123 \mathrm{mPa} \mathrm{s}$, with a good dispersion and won't produce "fish eye" when poured into the water after minor mixing. It doesn't produce "fish eye" and is more advantageous to the field of fracturing fluid system of site preparation after the chemical modification, of which can improve the dispersion of the powder under the premise of high dissolution rate.

Gel breaking performance: According to the petroleum industry standard SY/T 5107-2005 "water-based fracturing fluid performance evaluation method", on the use of ammonium persulfate and capsule acid gel breaker on $0.6 \%$ real time mixed fracturing fluid for the static breaking experiment $\left(90^{\circ} \mathrm{C}\right)$, the results in Table-2.

Experimental results show that, the amount of ammonium persulfate and capsule gel breaker can cause acid fracturing fluid gel breaking completely.

Repeated crosslinking performance: Fracturing fluid formula used in the experiment process is as follows: fast hydrating guar $+0.8 \%$ organic boron cross-linker (HTC-160 $+\mathrm{Na}_{2} \mathrm{CO}_{3}$ regulatory $\left.\mathrm{pH}=10\right)+$ capsule acid + additive. Fig. 2 is the fast hydrating guar fracturing fluid rheological curve measured under the condition of $100{ }^{\circ} \mathrm{C}, 170 \mathrm{~s}^{-1}$. Curve 1 is fracturing fluid prepared at the first time which sheared by 90 min and with a stable viscosity about $200 \mathrm{mPa}$ s and it doesn't affect the fracturing fluid, rheological properties in the process of fracturing after adding capsule acid. Curve 2 is the rheological curve which adding crushed capsule acid at $90{ }^{\circ} \mathrm{C}$, gel breaking $2 \mathrm{~h}$ first and then adding $\mathrm{Na}_{2} \mathrm{CO}_{3}$ to regulate $\mathrm{pH}$ value. As can be seen from the graph, the same after $90 \mathrm{~min}$ 
TABLE-1

MAIN PROPERTIES OF VARIOUS GUAR

\begin{tabular}{|c|c|c|c|c|c|c|}
\hline Name & $\begin{array}{l}\text { Moisture } \\
\text { content }(\%)\end{array}$ & $\begin{array}{c}\text { Apparent viscosity of } 0.6 \\
\% \text { solution (mPa s) }\end{array}$ & $\begin{array}{l}\text { Water insoluble } \\
\text { substance }(\%)\end{array}$ & $\begin{array}{l}\text { Residual content after } \\
200 \text { sieve mesh }(\%)\end{array}$ & Dispersity & $\mathrm{pH}$ \\
\hline JAG415 & 10.2 & 147 & 3.3 & 93 & Good & $6.5-7$ \\
\hline JAG418 & 10.0 & 142.5 & 3.5 & 93 & Good & $6.5-7$ \\
\hline Kunshan & 9.6 & 106.5 & 7.4 & 89 & Medium & 7 \\
\hline CMHPG & 11.6 & 81 & 8.9 & 90 & Poor & 7 \\
\hline AQULON & 12.1 & 93 & 8.5 & 90 & Medium & 7 \\
\hline FHG & 10.1 & 123 & 3.3 & 91 & Good & $6.5-7$ \\
\hline
\end{tabular}

Note: Good dispersion = A glass rod mild mixing can be dissolved, not caused 'fish eyes'; Dispersion medium = Mechanical stirrer low-speed stirring 5 min soluble, does not produce 'fish eye'; Poor dispersivity = Mechanical stirrer low-speed stirring 5 min soluble, to produce a small amount of 'fish eyes'; FHG = Fast hydrating guar.

TABLE-2

GEL BREAKING VISCOSITY OF GUAR FRACTURING FLUID

\begin{tabular}{ccc|cc}
\hline \multirow{2}{*}{$\begin{array}{c}\text { Time } \\
(\mathrm{min})\end{array}$} & \multicolumn{2}{c|}{$\begin{array}{c}\text { Gel breaking liquid apparent viscosity of } \\
\text { different ammonium persulfate dosage }(\mathrm{mPa} \mathrm{s})\end{array}$} & \multicolumn{2}{c}{$\begin{array}{c}\text { Gel breaking liquid apparent viscosity of } \\
\text { different capsule acid dosage (mPa s) }\end{array}$} \\
\cline { 2 - 5 } & $\begin{array}{c}\text { Mass fraction of } 0.03 \% \\
\text { ammonium persulfate }\end{array}$ & $\begin{array}{c}\text { Mass fraction of } 0.06 \% \\
\text { ammonium persulfate }\end{array}$ & $\begin{array}{c}\text { Mass fraction of } 0.03 \% \\
\text { capsule acid }\end{array}$ & $\begin{array}{c}\text { Mass fraction of } 0.06 \% \\
\text { capsule acid }\end{array}$ \\
\hline 120 & 11.1 & 9.1 & 14.7 & 9.4 \\
180 & 6.5 & 2.3 & 8.8 & 4.5 \\
\hline
\end{tabular}

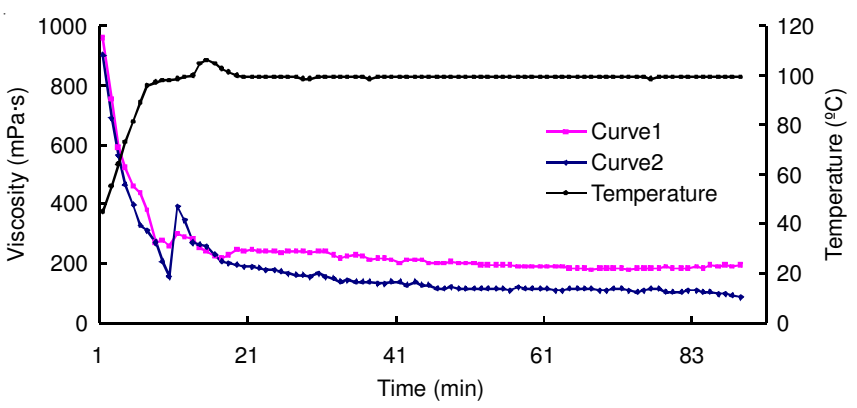

Fig. 2. Rheological curve of fast hydrating guar fracturing fluid

shear, viscosity of fracturing fluid is maintained at $85 \mathrm{mPa} \mathrm{s}$, which indicates that this fast hydrating guar fracturing fluid gel breaking can be reutilized after the adjustment of $\mathrm{pH}$ value.

The laboratory experiment shows that molecular chain can't be damaged, by adjusting the $\mathrm{pH}$ value of the fast hydrating guar fracturing fluid system with non degradable gel breaking, which can realize the reutilization of fracturing fluid and flow-back liquid can be reused, which is good for economy, energy saving and environment protection.

\section{Conclusion}

After the properties contrast of fast hydrating guar and several modified guar and research on the mechanism of the fast hydrating guar dissolution, we compounded the fracturing fluid which used the fast hydrating guar as a thickening agent and also carried on the repeated cross-linking performance test. The experimental results show that fast hydrating guar is an excellent performance of fracturing fluid thickening agent, which has a higher viscosity, dissolved rapidly, good dispersion, good cross-linking stability, gel breaking and other advantages and also has certain renewable performance and a wide application prospect. This technology can improve the site fracturing fluid preparing efficiency, which can save production cost and reduce the pollution to the environment with a certain development potential. But the cross-linking technology is still not mature, because the optional slow release acid is less and this is the next research direction.

\section{ACKNOWLEDGEMENTS}

This work was supported by the National Major Special Projects of China(Grant No. 2011ZX05051).

\section{REFERENCES}

1. M.Z. Li, X.Q. Liu, Z.S. Tang, Y. Wang, C.M. Zhou and J.H. Liu, Oilfield Chem., 19, 89 (2002).

2. B.S. Guan, J. Liu, X.Q. Zhou, X.-J. Xue, Y.-M. Zhang and X. Xie, Oilfield Chem., 25, 126 (2008).

3. J. Wang, B.A. Yi and S.Y. Yuan, Chem. Eng. Oil Gas, 40, 66 (2011).

4. S. Centurion and M.S. Alcantar, SPE59245 (2000).

5. L. Ding, H.Y. Lv, W. Zhao, J. Wu and L. Lu, Chem. Eng. Oil Gas, 39, 316 (2010).

6. Y.C. Chen, S. Zhang, X.S. Wei, X.-S. Liu and H.-T. Zhao, Nat. Gas Ind., 30, 30 (2010).

7. Z.F. Zhuang, X. Zhao, J. Li, R.-Q. Zhao and C. Yang, Oilfield Chem., 26, 139 (2009).

8. R.Z. Jiang, T.X. Jiang and Y.L. Wang, Oil Drill. Prod. Technol., 26, 52 (2004).

9. C.Y. Li, Y.D. Wang and Q.X. Zeng, Inner Mongolia Petrochem. Ind., 5, 8 (2011).

10. J. Peles, R.W. Wardlow, G. Cox, W. Haley, R. Dusterhoft, H.G. Walters and J. Weaver, SPE77746 (2002).

11. X.J. Zheng, J.H. Su, C.M. Xu, et al., Appl. Chem. Ind., 38, 1623 (2009). 